\title{
On Researching the Vehicle Monitoring Platform Based on Spatio-Temporal Indexing Mechanism
}

\author{
http://dx.doi.org/10.3991/ijoe.v9iS7.3191 \\ ZHAN Shaobin, LIANG Yongsheng \\ Shenzhen Institute of Information Technology, Shenzhen, China \\ Graduate School at Shenzhen, Tsinghua University Shenzhen,China \\ Gaoxin Modern Shenzhen,China
}

\begin{abstract}
It's the research hotspots that Spatio-Temporal Indexing Mechanism(STIM) in GIS, especially in vehicles monitoring and scheduling field. Then, it's more important how to unite the time and space information, improve the query efficiency. At the beginning of design system, constructing Floating Model Indexing Mechanism(FMIM). It established a three-layers of data query structure, which can guarantee the effective Spatio-Temporal combination. According running track of vehicles, vehicle and operation is proposed based on t-Time Dual-mode Index Mechanism and T-Period Vehicle Monitoring Index Mechanism. The related technical has been commercialized, it can efficiently improve query efficiency. The regulatory platform running well.
\end{abstract}

Index Terms-Spatio-Temporal Indexing Mechanism; Floating Model Indexing Mechanism; Dual-mode Index Mechanism

\section{INTRODUCTION}

With the development of positioning technology(Such as GPS, mobile phone location), more and more applications based on location data have produced(such as LBS, vehicle monitoring system etc.). It is the most basic of Space and time, the most important attribute in the field[1-3]. Compared with traditional databases, spatiotemporal database has a much larger amount of data. It is one of temporal database research priorities that establishing an effective spatio-temporal indexing to improve the efficiency of various types of space-time queries in this paper.

The traditional GIS system of less precision of time, most are in units of years or months, such as pipeline system, geological features system etc[4-6]. In this case, adding the time information to the index structure node[7], or creating overlap index structure all can create the user needs to complete the operation[8]. But against real-time strongly operating system(such as vehicle control, fire safety, traffic dispatched, police support systems etc, they are all time and space interaction platform in hours, minutes and seconds), the amount of time information is large, called frequency is high, and spatial information exchange is much, establishing an effective indexing mechanism is necessary[9]. The combination of a variety of index structures is the main trend in the actual design of spatial and temporal database system[10]. It is the main challenges facing the design of system that choose which of several index structures and how to organically combine them.

\section{Construction of Floating Model Spatio- TEMPORAL INDEXING MECHANISM}

First of all, in order to ensure the vehicle time information combined with spatial location information, creating geographic information database model based on space-time index and three-tier data query structure integrates real-time vehicle information and geographic databases. The first two layers model are based on own property of GIS system and the two-way link structure of spatial location, using a common GeoDatabase management, through spatial data engine to realize the interoperability, the introduction of the concept of time and space in the third layer, the technology can realize the two-way link of temporal and properties. In order to ensure that the attribute information and spatial information do not produce redundant, based on combining the traditional spatio-temporal model, established spatio-temporal index table as a link approach in the relational database, three-tier data can be combined through the attribute information and be coordinated operation.

Common GIS softwares such as: ArcGIS series of ESRI, SuperMap Software Co. Ltd.8-9 etc. Based on the link of spatio-temporal information and attribute information, we have established spatio-temporal filtering and floating model to sort queries. Figure 1 shown.

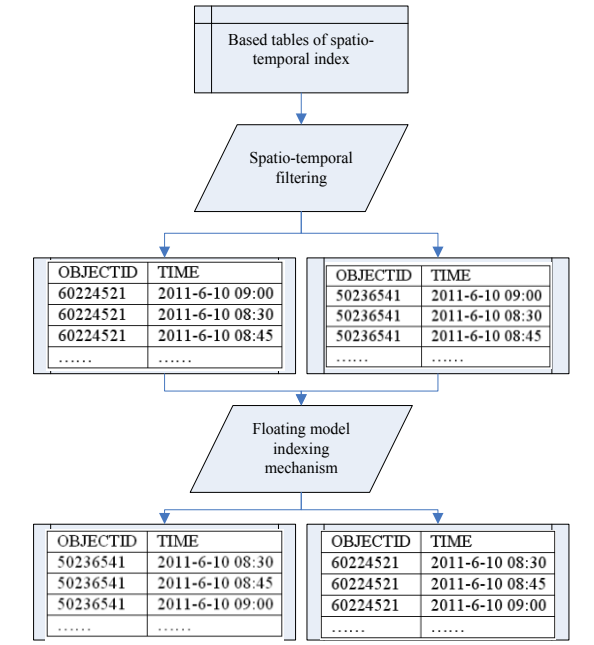

Figure 1. Spatio-temporal index part of the logic model

Through a unified of eight coding all the spatiotemporal information and data carry on spatio-temporal filtering, divided into several groups of view which organizes structurally to spatial and temporal information 
of a single ID. Using self-built floating model sort, model is similar to floating objects in water, light floating in the above, turn down the density becomes larger. In database, the difference is using time to replace the density, time front in the data view top. According to the establishment of spatio-temporal index table, it can be completed very well that the effective integration of time information, spatial information and attribute information. And on the basis of the existing simple model, we can expect the situation of vehicles for some time.

\section{DuAL-MODEL INDEXING Mechanism BASED ON T TIME POINT}

When the vehicle is running, taking into account the communication costs and the requirements of GPS satellite positioning, we use dual-mode co-location of GPS + GPSOne, in-vehicle terminal transfer location information using fixed time or frequency point, after processing the information obtained information for the fixed time point. The demand for dual-mode positioning improves extracting information on the proper time. First, it ensures the switching criteria of ways to position, then adopts a variant structure of R-tree to execute spatiotemporal index, in this respect, we define the following criteriaes.

GUIDELINES 1. Inertia criterion: $r=\mathrm{Z}(\mathrm{d}, \mathrm{p}, \mathrm{s}) \mathrm{U} \mathrm{B}, \mathrm{Z}$ as the adequacy of mode conversion is a function of $d$ as collecting information and data, $\mathrm{p}$ as being tested procedures and $\mathrm{s}$ as function statute. It means that program $p$ relative to $s$ completes the function degree of $d$. Variable B is a function of the range in the real interval $[0,1]$. $U$ shows the value of $r$ is subject to both the impact of $\mathrm{Z}$ and $\mathrm{B}$. The value of $\mathrm{B}$ can decide directly the result of $r$. When $B$ is taken as the critical value of 0 or $1, r$ is also direct access to 0 or 1 .The greater $r$ the higher sufficient degree.

GUIDELINES 2. Efficiency criterion: Criterion $\mathrm{C}$ is a predication defined in the $\mathrm{T} \times \mathrm{D} \times \mathrm{S}$, is $\mathrm{C}: \mathrm{T} \times \mathrm{D} \times \mathrm{S} \rightarrow\{$ true, false $\} . \mathrm{C}(\mathrm{t}, \mathrm{d}, \mathrm{s})=$ true means access to location information data set $\mathrm{d}$, for function statute $\mathrm{s}$, within the allowable range in time $t$, to complete the program is fully. $\mathrm{C}(\mathrm{t}, \mathrm{d}, \mathrm{s})=$ false means inadequate, need dual-mode switch.

GUIDELINES 2 SUPPLEMENTARY: Criterion $\mathrm{C}$ is a predication which is the projection of the economic range in $\mathrm{V}[0,1]$ defined in the $\mathrm{T} \times \mathrm{D} \times \mathrm{S}$, is $\mathrm{C}(\mathrm{B} 0,1)$ :

$\mathrm{T} \times \mathrm{D} \times \mathrm{S} \rightarrow \mathrm{V}$, if and only if the brake value $\mathrm{B}$ is true, $\mathrm{C} \rightarrow$ \{true, false .

Supplementary of guidelines 2 has joined the external decision variable $B$, supported mandatory changes in the external interface, at the same time changed the range of $\mathrm{C}$ into the projection of the economy's range $\mathrm{V}$ to determine its true or false, further improved the practicability of the guidelines 2. Dual-mode indexing mechanism based on $t$ time point can reduce blind spots and improve accuracy.

Improvement in the conversion standard R-tree structure as follows:

1.in every level of the tree ,minimize the area of the bounding rectangle;

2.minimize the dead space of the bounding rectangle; 3.reduce the binding rectangle intersection of each level.

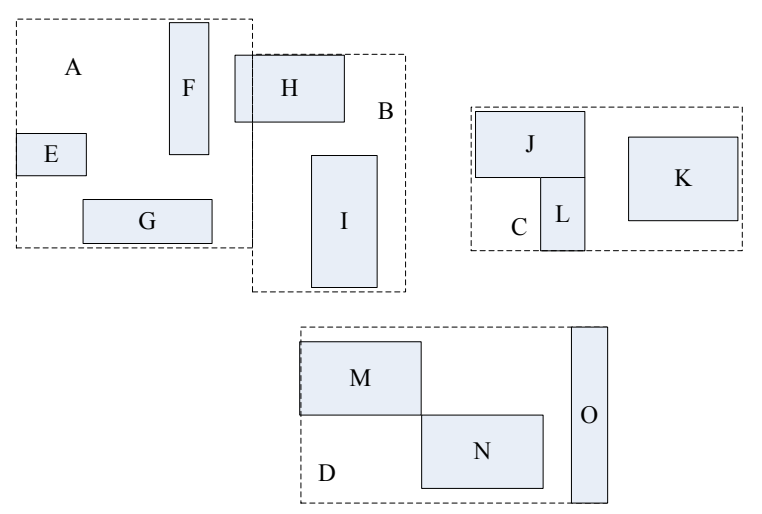

Figure 2. Original organization

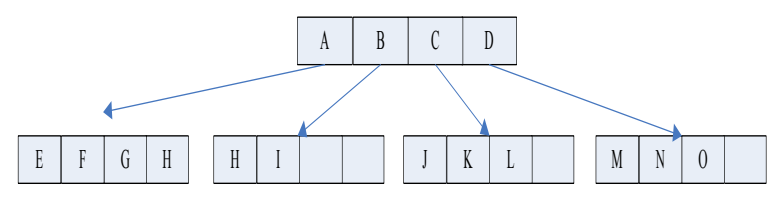

Figure 3. R-tree organization

\section{INDEXING MECHANISM RESEARCH BASED ON T TIME PERIOD}

Time information itself has continuity and sequential, When the sample point information for the time period, does not join in query engine mechanism as dot information. Video monitoring of vehicle operation obtained in the service center exists such circumstances of time period query, for this reason, we use segmentation algorithm. Using 4R-tree operations according to nodes' different termination value of the effective time and the transaction time, corresponding records can be updated.

The indexing algorithm is as follows:

$$
\begin{aligned}
& \operatorname{search}\left(T T^{\rightarrow}, T T^{\leftarrow}, V T^{\rightarrow}, V T^{\leftarrow},\right)= \\
& \operatorname{search} R_{1}\left(0, T T^{\leftarrow}, 0, V T^{\leftarrow}\right) \cup \\
& \operatorname{search} R_{2}\left(0, T T^{\leftarrow}, V T^{\rightarrow}, V T^{\leftarrow}\right) \bigcup \\
& \operatorname{search}_{3}\left(\max \left(T T^{\rightarrow}, T T^{\leftarrow}\right), T T^{\leftarrow}, 0, V T^{\leftarrow}\right) \cup \\
& \operatorname{searchR}_{4}\left(T T^{\rightarrow}, T T^{\leftarrow}, V T^{\rightarrow}, V T^{\leftarrow}\right) \\
& \text { if } T T^{\rightarrow} \neq C T \wedge V T^{\leftarrow} \geq V T^{\rightarrow} \text {; } \\
& \operatorname{search} R_{1}\left(0, T T^{\leftarrow}, 0, V T^{\leftarrow}\right) \bigcup \\
& \operatorname{searchR}_{2}\left(0, T T^{\leftarrow}, V T^{\rightarrow}, V T^{\leftarrow}\right) \bigcup \\
& \operatorname{search}_{3}\left(\max \left(T T^{\rightarrow}, T T^{\leftarrow}\right), T T^{\leftarrow}, 0, V T^{\leftarrow}\right) \cup \\
& \operatorname{searchR}_{4}\left(T T^{\rightarrow}, T T^{\leftarrow}, V T^{\rightarrow}, V T^{\leftarrow}\right) \\
& \text { if } T T^{\rightarrow} \neq C T \wedge V T^{\leftarrow} \geq V T^{\rightarrow}<V T^{\rightarrow} \text {; } \\
& \operatorname{search} R_{1}\left(0, T T^{\leftarrow}, 0, V T^{\leftarrow}\right) \bigcup \\
& \operatorname{search} R_{2}\left(0, T T^{\leftarrow}, V T^{\rightarrow}, V T^{\leftarrow}\right) \bigcup \\
& \text { if } T T^{\rightarrow}=C T \wedge\left[V T^{\rightarrow}, V T^{\leftarrow}\right] \neq\left[t_{\min }, t_{\max }\right] \wedge T T^{\leftarrow} \geq V T^{\rightarrow} \text {; } \\
& \operatorname{search} R_{2}\left(0, T T^{\leftarrow}, V T^{\rightarrow}, V T^{\leftarrow}\right) \\
& \text { if } T T^{\rightarrow}=C T \wedge\left[V T^{\rightarrow}, V T^{\leftarrow}\right] \neq\left[t_{\text {min }}, t_{\text {max }}\right] \wedge T T^{\leftarrow}<V T^{\rightarrow} \text {; } \\
& R_{1} \cup R_{2} \text { if } T T^{\rightarrow}=C T \wedge\left\lfloor V T^{\rightarrow}, V T^{\leftarrow}\right\rfloor=\left[t_{\min }, t_{\max }\right\rfloor
\end{aligned}
$$


4R-tree index compose with four R-tree, the essence is to transform the different arguments into different fixed values through various transformations. Existing GR-tree are mapped, get R-tree of 4 different forms, and then query based on the traditional R-tree index. Thereby the GR-tree index of two temporal data can be completed, and can adapt to the current DBMS very well.

\section{EFFECt Detection OF SYSTEM}

Based on vehicle monitoring platform, we have tested spatio-temporal indexing mechanism for $\mathrm{t}$ time point and $\mathrm{T}$ time period. Actual data show that it can improve the query efficiency and achieve the corresponding functions.

\section{A. Application of spatio-temporal indexing mechanism based on time point}

Experimental data are two vector data of different state and same scale. First, two different state data are related according to the changes of entity's location and properties, created spatio-temporal index. Then the version data produced is inquired at this time point in the light of the specified time.

Spatio-temporal query efficiency analysis is a query efficiency analysis based on the different number of time points queried in figure 4. Experiments show that:

- Query speed has been improved greatly according to compare spatio-temporal query using $\mathrm{S}-\mathrm{T}$ index with untapped S-T index based on t time point.

- With the query time points increased, query speed is improved obviously.

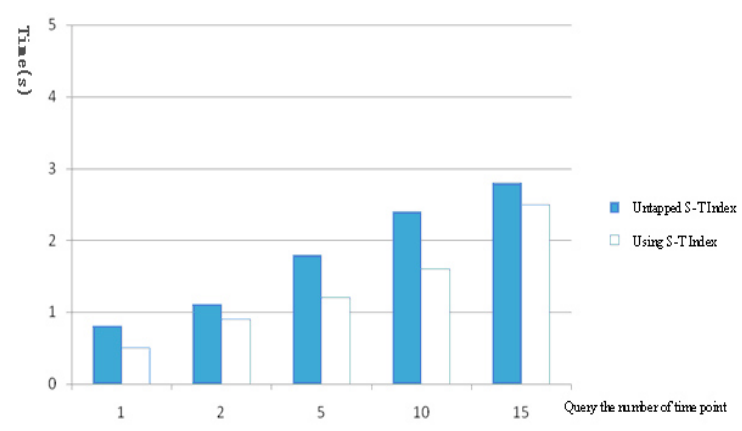

Figure 4. Spatio-temporal query efficiency analysis

Figure5 shows the state analysis of hardware during spatio-temporal indexing, operating results fluctuate within the normal range, the lowest point represents the effect of accessing data. Based on system operation of spatio-temporal index, maximum is $61.2 \mathrm{MB} / \mathrm{S}$, completely within the normal processing of the computer. CPU average around $2 \%$, explains that the proposed algorithms and techniques to achieve better query than the traditional method results in the case of less demanding of hardware resources in this paper.
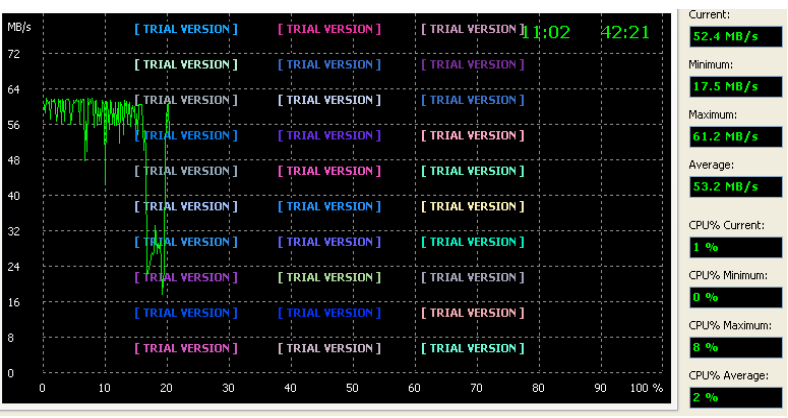

Figure 5. Analysis of hardware status

\section{B. Spatio-temporal data model of moving target trajectory}

The application of index mechanism based on time period can be expressed the spatial information visualization as time for clue. Spatio-temporal visualization provides dynamic simulation of moving objects visualization in space, including the historical review and future prediction etc, will fundamentally change the existing theory system of spatial information visualization.

Below is that the vehicle dynamic control system running interface which we have developed, figure 6 is shown, FAW-Volkswagen BORA, speed of $30 \mathrm{~km} / \mathrm{h}$, starting from the junction of Longzhu Four Road and Longzhu Avenue, traveling southeast along Longzhu Avenue, exiting from Vehicle management exit, traveling about $1.8 \mathrm{~km}$, taking 4 minutes, to the red track centerline as the standard, the system can monitor vehicle current position, playback history position and predict the future.

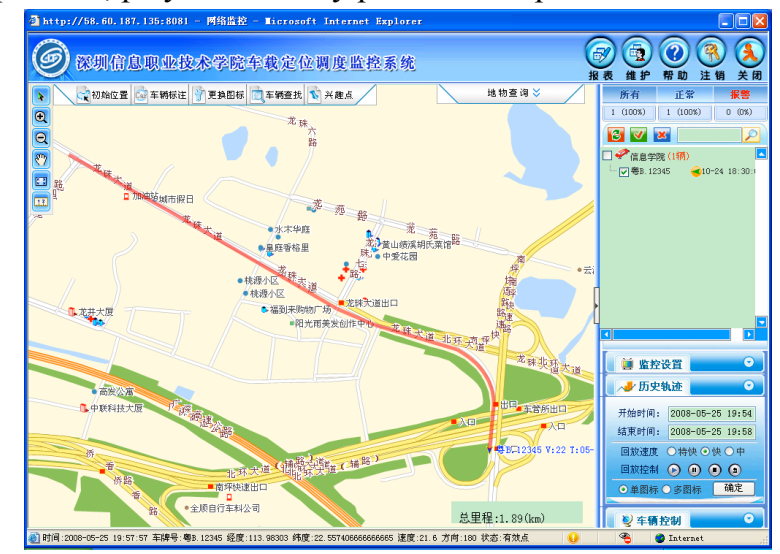

Figure 6. vehicle dynamic control system

\section{Running effect detection}

\section{Response time}

Monitoring of the system is carried out by the two modules to existing technologies, GPS positioning returned can be controlled in less than one second, GPSOne relative positioning longer, generally about 30 to 50 seconds, but also according to the signal strength of the environment in which to decide. Based on the space-time indexing mechanism to the response time of the experiments, the following measurements.

\subsection{Vehicles in the urban}

Purpose of the experiment: measure the response time in the process of moving 
Experimental sections: from the main entrance of the SZIIT to the Science Museum, about three kilometers, 4 stops traffic lights.

Experimental vehicles: BORA. Speed: $60 \mathrm{~km} / \mathrm{h}$

Experimental Method: First, correct two clock. Then, one on the vehicle record the GPS receive time, another record the time which get from Spatio-Temporal database. Step is ten seconds. The key experimental records are shown in Table 1.

TABLE I.

RESPONSE TIME IN THE URBAN

\begin{tabular}{|l|l|l|l|l|l|l|}
\hline $\begin{array}{c}\text { Typical } \\
\text { Objects }\end{array}$ & $\begin{array}{c}\text { BaGua } \\
\text { 3 Road }\end{array}$ & Stadium & $\begin{array}{c}\text { SunGang } \\
\text { West } \\
\text { Road }\end{array}$ & $\begin{array}{c}\text { Sichuan } \\
\text { Building }\end{array}$ & $\begin{array}{c}\text { Municipal } \\
\text { People's } \\
\text { Congress }\end{array}$ & $\begin{array}{c}\text { Shennan } \\
\text { Road }\end{array}$ \\
\hline $\begin{array}{l}\text { Recording } \\
\text { time }\end{array}$ & $15: 25: 10$ & $15: 27: 23$ & $15: 28: 51$ & $15: 35: 47$ & $15: 36: 21$ & $15: 39: 07$ \\
\hline Positioning & $15: 25: 9$ & $15: 27: 23$ & $15: 28: 53$ & $15: 35: 47$ & $15: 36: 21$ & $15: 39: 05$ \\
\hline Error & $+0: 00: 01$ & 0 & $-0: 00: 02$ & 0 & 0 & $+0: 00: 02$ \\
\hline
\end{tabular}

\section{2 vehicles in campus}

In the campus, from the 1 st Teaching building to laboratory ,It's about $500 \mathrm{~m}$, the time which get from Spatio-Temporal database respectively is equal the receive time, error is completely negligible.

\section{Massive data retrieval}

In order to detect the effect of Spatio-Temporal Indexing Mechanism for massive data retrieval, we try to increase the remote sensing image, multi temporal vehicle monitoring data and video. Then, we obtained the relationship between the retrieval time and the amount of data. It's shown in figure 7.

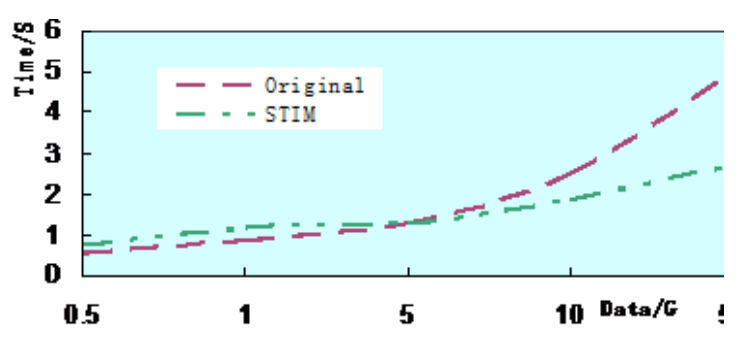

Figure 7. relationship between retrieval time and data

In figure 7 , original expressed the common relational database retrieval mechanism, STIM expressed the SpatioTemporal Indexing Mechanism. The abscissa is the amount of data in $\mathrm{GB}(\mathrm{G})$, and the ordinate represents the response time in seconds(S). When the amount of data is smaller, the time of traditional retrieval mechanism is shorter than the Spatio-Temporal Indexing Mechanism. Because the Spatio-Temporal Indexing Mechanism includes more algorithms. When data increases, the time delay generated by the algorithm is negligible. So, it's faster than original. At the same time, when the amount of data is small, temporal and spatial indexing mechanism is relatively slow, But the user can ignore the time.

\section{CONCLUSIONS}

On the basis of constructing floating model indexing mechanism, contrary to vehicle tracks and video surveillance, based on t-Time Dual-mode Index
Mechanism and T-Period Vehicle Monitoring Index Mechanism have been discussed. At the same time, On the basis of the relevant theoretical algorithm, we have developed vehicle location control and supervision system based on B / S structure, which can understand the vehicle trajectory and monitor the situation inside the vehicle, related products through its promotion of the industrialization, have been sold thousands of Software and hardware units, running in good condition.

\section{ACKNOWLEDGMENTS}

1. The research was sponsored by the Natural Science Foundation of Guangdong Province (S2012010009419) and The research was sponsored by Shenzhen strategic emerging industry development funds (JCYJ20120821162230172).

\section{REFERENCES}

[1] Jain, Vani, Spatio-temporal querying of video content using SQL for quantizable video databases, Journal of Multimedia, v 4, n 4, p 215-227, August 2009.

[2] Huang, Yuan-Ko Continuous K-Nearest neighbor query for moving objects with uncertain velocity, Information Systems, v 34, n 4-5, p 415-437, July 2009. http://dx.doi.org/10.1016/j.is.20 09.01 .001

[3] Lindeberg,Tony. Generalized gaussian scale-space axiomatics comprising linear scale-space, affine scale-space and spatiotemporal scale-space.Journal of Mathematical Imaging and Vision, v 40, n 1, p 36-81, May 2011. http://dx.doi.org/10.1007/ s10851-010-0242-2

[4] http://www.esri.com/ 2011-8-15.

[5] Ribaric, Slobodan; Hrkac, Tomislav. A model of fuzzy spatiotemporal knowledge representation and reasoning based on highlevel Petri nets.Information Systems, v 37, n 3, p 238-256, May 2012. http://dx.doi.org/10.1016/j.is.2011.09.010

[6] MacIejewski, Ross ,A visual analytics approach to understanding spatiotemporal hotspots,IEEE Transactions on Visualization and Computer Graphics, v 16, n 2, p 205-220, March-April 2010. http://dx.doi.org/10.1109/TVCG.2009.100

[7] Zhan Shaobin, Liang Yongsheng, The method of mobile terminal zero drift and single-mode \& dual-mode localization, Patent Application No. 200810217455.3.

[8] SB Zhan, YS Liang,M Nie. Researching on Smoothly Moving algorithm of GPS \& GPSOne Switching Pattern. Proceedings of International Conference on Information Technology and Environment System Sciences. 2008 : 1089 1094.

[9] Zhan Shaobin. Lu xin ;Gao yuefang.System Design of Number Inquiry Software Basing on J2ME. Proceedings of 2010 Second International Conference on Computer Modeling and Simulation.2010.1.22:88 92.

[10] Zhan shaobin, Liang yongsheng, Gao Yuefang, Jin houzhong. On Researching the Key Algorithm of Dual-mode Vehicle Positioning System. Proceedings of the 3rd IEEE International Conference on Computer Science and Information Technology. 2010.7:659 662.

[11] Jeung, Hoyoung Convoy queries in spatio-temporal databases, International Conference on Data Engineering, p 1457-1459, 2008.

[12] Seshadrinathan, Kalpana, Motion tuned spatio-temporal quality assessment of natural videos, IEEE Transactions on Image Processing, v 19, n 2, p 335-350, February 2010. http://dx.doi.org/10.1109/TIP.2009.2034992

\section{AUTHORS}

Zhan shaobin received his Ph.D. in GIS engineering from Jilin University China. His research direction is Grid computing and software development \& application. He 
had attended National Natural Sciences Foundation of China (NSFC) (no.40471086), the National High Technology Research and Development Program ("863"Program) of China (2006AA12Z102). In 2007, he came to Institute of Information Technology in Shenzhen, where he continued his research. Now Dr. Zhan has published more than 30 journal papers and co-authored several government reports.(13266860452@163.com.cn)

Prof.Liang yongsheng received the B.S. degree from Shanxi University of Science and Technology, Xi'an, China, and the PH.D. degree from Harbin Institute of Technology, Harbin, China, in 1993 and 1999 respectively. He completed the post doctoral research in information and communication engineering Station of Harbin Institute of Technology, Harbin, China, in 2005. $\mathrm{He}$ has been a professor at Shenzhen Institute of Information Technology, Shenzhen, China. He is now a professor at the School of Information Engineering of Shenzhen University, Shenzhen, China. He is an IEEE ComSoc member. His research interests include computer network and data communication, signal processing and pattern recognition.(liangys@sziit.com.cn)

Submitted, 14 August 2013. Published as resubmitted by the authors on 22 October 2013. 